\title{
Relationships between hardness, elastic modulus, and the work of indentation
}

\author{
Yang-Tse Cheng ${ }^{\text {a) }}$ \\ Department of Physics and Physical Chemistry, General Motors Research and Development Center, \\ Warren, Michigan 48090 \\ Che-Min Cheng ${ }^{\text {b) }}$ \\ Laboratory for Non-Linear Mechanics of Continuous Media, Institute of Mechanics, \\ Chinese Academy of Sciences, Beijing 100080, People's Republic of China
}

(Received 12 February 1998; accepted for publication 30 May 1998)

\begin{abstract}
The work done during indentation is examined using dimensional analysis and finite element calculations for conical indentation in elastic-plastic solids with work hardening. An approximate relationship between the ratio of hardness to elastic modulus and the ratio of irreversible work to total work in indentation is found. Consequently, the ratio of hardness to elastic modulus may be obtained directly from measuring the work of indentation. Together with a well-known relationship between elastic modulus, initial unloading slope, and contact area, a new method is then suggested for estimating the hardness and modulus of solids using instrumented indentation with conical or pyramidal indenters. (c) 1998 American Institute of Physics. [S0003-6951(98)02931-3]
\end{abstract}

Indentation experiments have been performed for nearly one hundred years for measuring the hardness of materials. ${ }^{1}$ Recent years have seen increased interest in indentation because of the significant improvement in indentation equipment and the need for measuring the mechanical properties of materials on small scales. It is now possible to monitor, with high precision and accuracy, both the load and displacement of an indenter during indentation experiments in the respective micro-Newton and nanometer ranges. ${ }^{2-4}$ Using these instrumented indentation techniques, the hardness and Young's modulus may be obtained from the peak load and the initial slope of the unloading curves by the method of Oliver and Pharr ${ }^{5}$ or that of Doerner and Nix. ${ }^{6}$ Both methods, however, depend on estimating contact area under load, which is sometimes difficult, especially when "piling-up" occurs.

Using a scaling approach to indentation problems developed recently, ${ }^{7,8}$ we reveal, in this letter, an approximate relationship between the ratio of hardness to elastic modulus and the ratio of irreversible work to total work in indentation. Consequently, the ratio of hardness to elastic modulus may be obtained directly from measuring the work of indentation. Together with a well-known relationship between elastic modulus, initial unloading slope, and contact area, a new method is then suggested for estimating the hardness and modulus of solids using instrumented indentation with conical or pyramidal indenters.

We consider a three-dimensional, rigid, conical indenter of a given half angle, $\theta$, indenting normally into a homogeneous elastic-plastic solid with work hardening. The friction coefficient at the contact surface between the indenter and the solid is assumed zero. Below the initial yield stress, $Y$, the solid is elastic and is characterized by the Young's modulus, E, and Poisson's ratio, $\nu$. After yielding, stress-

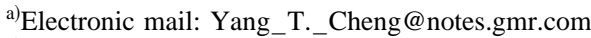

${ }^{b)}$ Electronic mail: zhengzm@LNM.imech.ac.cn
}

strain $(\sigma-\epsilon)$ curves of the solids under uniaxial tension are assumed to be

$$
\sigma=K \epsilon^{n}, \text { for } \epsilon \geqslant Y / E,
$$

where $K$ is the strength coefficient and $n$ is the workhardening exponent. ${ }^{9}$ To ensure continuity, $K=Y(E / Y)^{n}$. Consequently, either $E, Y$, and $K$ or $E, Y$, and $n$ are sufficient to describe the stress-strain relationship. We use the latter set of parameters extensively in the following discussions. When $n$ is zero, Eq. (1) becomes the model for elasticperfectly plastic solids. For most metals, $n$ has a value between 0.10 and $0.50 .^{10}$

During loading, the force, $F$, on the indenter can be written, according to dimensional analysis, as ${ }^{7,8}$

$$
F=E h^{2} \Pi_{\alpha}(Y / E, \nu, n, \theta),
$$

where $\Pi_{\alpha}$ is a dimensionless function of four dimensionless parameters $Y / E, \nu, n$, and the indenter half angle $\theta$. From Eq. (2), the total work done by the indenter, $W_{\text {tot }}$, to cause elastic and plastic deformation when the indenter reaches a maximum depth, $h_{m}$, is given by

$$
W_{\mathrm{tot}}=\int_{0}^{h_{m}} F d h=\frac{E h_{m}^{3}}{3} \Pi_{\alpha}\left(\frac{Y}{E}, \nu, n, \theta\right) .
$$

Thus, the force and work are proportional to $h_{m}^{2}$ and $h_{m}^{3}$, respectively.

Since unloading takes place after loading during which the indenter reaches the maximum depth, $h_{m}$, the force, $F$, on the indenter can be written, according to dimensional analysis, as ${ }^{7,8}$

$$
F=E h^{2} \Pi_{\gamma}\left(\frac{Y}{E}, \frac{h}{h_{m}}, \nu, n, \theta\right),
$$

where $\Pi_{\gamma}$ is a dimensionless function of five dimensionless parameters, $Y / E, h / h_{m}, \nu, n$, and $\theta$. The force is, in general, no longer proportional to the square of the indenter displacement. It can, in principle, depend on $h / h_{m}$ through the di- 


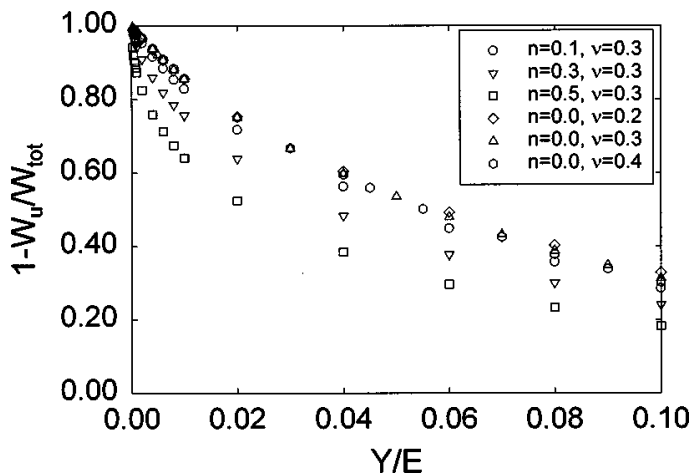

FIG. 1. Scaling relationship for $\left(W_{\text {tot }}-W_{u}\right) / W_{\text {tot }}=\Pi_{w}(Y / E, \nu, n, \theta)$ for $\theta$ $=68^{\circ}$.

mensionless function $\Pi_{\gamma}$. Furthermore, the final depth, $h_{f}$, at which the force on the indenter first becomes zero during unloading, can be obtained by solving

$$
F=E h_{m}^{2} \Pi_{\gamma}\left(\frac{Y}{E}, \frac{h_{f}}{h_{m}}, \nu, n, \theta\right)=0 .
$$

Clearly, $h_{f} / h_{m}$ is a function of $Y / E, \nu, n$, and $\theta$.

The work done by the solid to the indenter during unloading, $W_{u}$, can now be expressed as

$$
\begin{aligned}
W_{u}=\int_{h_{f}}^{h_{m}} F d h & =E h_{m}^{3} \int_{h_{f} / h_{m}}^{1} x^{2} \Pi_{\gamma}\left(\frac{Y}{E}, x, \nu, n, \theta\right) d x \\
& \equiv E h_{m}^{3} \Pi_{u}\left(\frac{Y}{E}, \nu, n, \theta\right),
\end{aligned}
$$

where the integral, $\int_{h_{f} / h_{m}}^{1} x^{2} \Pi_{\gamma}(Y / E, x, \nu, n, \theta) d x$, is obviously a dimensionless function, $\Pi_{u}$, of $Y / E, \nu, n$, and $\theta$. Consequently, the work done on the indenter during unloading is also proportional to $h_{m}^{3}$. The ratio of irreversible work to total work for a complete loading-unloading cycle, 1 $-W_{u} / W_{\text {tot }}$, can be written as

$$
\frac{W_{\mathrm{tot}}-W_{u}}{W_{\mathrm{tot}}}=1-3 \frac{\Pi_{u}(Y / E, \nu, n, \theta)}{\Pi_{a}(Y / E, \nu, n, \theta)} \equiv \Pi_{w}\left(\frac{Y}{E}, \nu, n, \theta\right) .
$$

This ratio is, therefore, independent of $h_{m}$.

Because there is, as yet, no analytical solution to the problem of conical indentation in elastic-plastic solids, we chose to use finite element calculations to evaluate the dimensionless function, $\Pi_{w}$. Since there are only a few indenter angles of experimental interest, we fix $\theta=68^{\circ}$ as in early studies (e.g., Ref. 11) and evaluate the dependence on $Y / E, \nu$, and $n$ to reveal the essential physics of the work of indentation. The details of the finite element model have been given previously. ${ }^{12}$ In Fig. 1 the function $\Pi_{w}$ is evaluated for many values of $Y / E, \nu$, and $n$. Consistent with common experiences, the ratio of irreversible work to total work decreases with increasing $Y / E$ and $n$, i.e., when the solid is more "elastic.",

In a previous study, ${ }^{8}$ we have examined the functional dependence of hardness $H$, defined as the force divided by the projected contact area under full load, on $E, \nu, Y$, and $n$. Using Eq. (18) in Ref. 8, the ratio of hardness to Young's modulus, $E$, can be written as modulus, $E$, can be written as $\left.-W_{u}\right) / W_{\text {tot }}<1.0$ and (b) details for $0.9<\left(W_{\text {tot }}-W_{u}\right) / W_{\text {tot }}<1.0$.
Downloaded 06 Jun 2008 to 159.226 .100 .195$. Redistribution subject to AlP license or copyright; see http://apl.aip.org/apl/cop

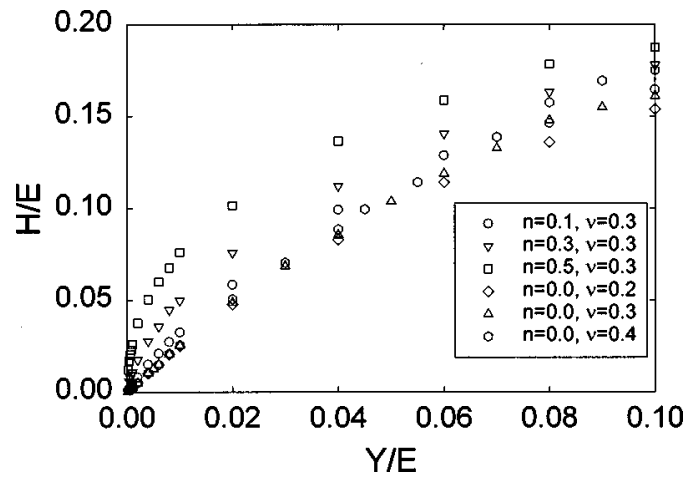

FIG. 2. Scaling relationship for $H / E=\Pi_{h}(Y / E, \nu, n, \theta)$ for $\theta=68^{\circ}$.

$$
\frac{H}{E}=\Pi_{h}\left(\frac{Y}{E}, \nu, n, \theta\right),
$$

where $\Pi_{h}$ is a dimensionless function of $Y / E, \nu, n$, and $\theta$. The value of $\Pi_{h}$ calculated using the same finite element method is shown in Fig. 2. As expected, the value of $H / E$ increases with increasing $Y / E$ and $n$.

While much of the physics of hardness and irreversible work of indentation are contained in Eqs. (6) and (7), it is instructive to investigate the relationship between $H / E^{*}$ and $1-W_{u} / W_{\text {tot }}$, where $E^{*}=E /\left(1-\nu^{2}\right)$. In Fig. 3, we replot all the data points shown in Figs. 1 and 2 in the form of $H / E^{*}$ vs $1-W_{u} / W_{\text {tot }}$. It is evident from Fig. 3 that all the data points lie approximately on a single curve. Consequently, we may write

$$
\frac{H}{E^{*}} \approx \Pi_{\theta}\left(\frac{W_{\mathrm{tot}}-W_{u}}{W_{\mathrm{tot}}}\right) .
$$
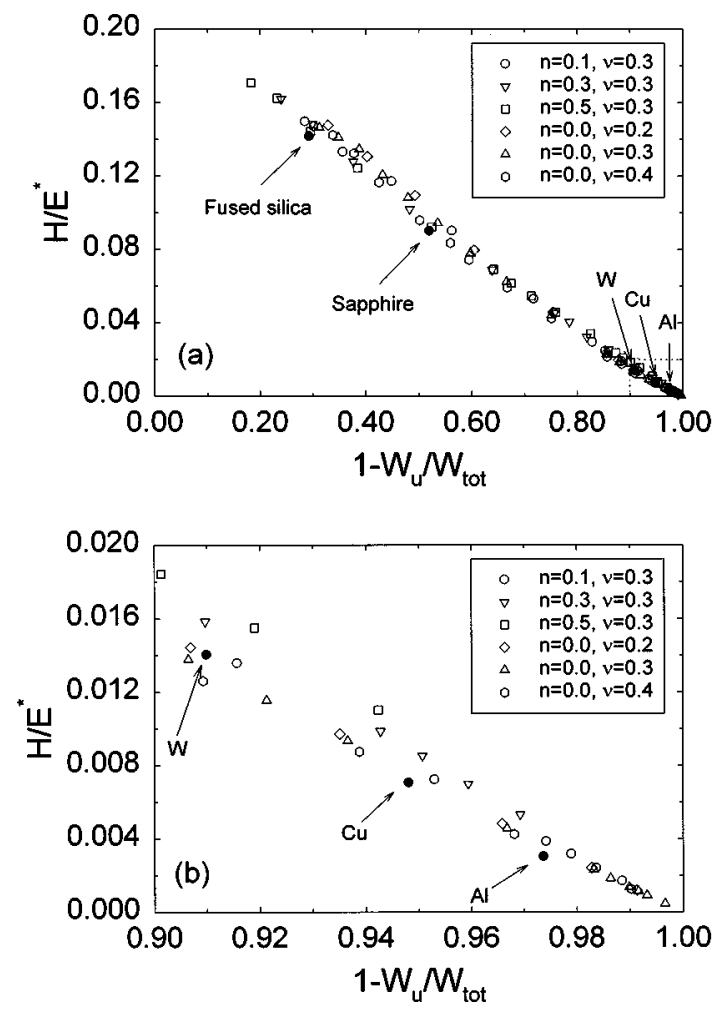

FIG. 3. Relationship between $H / E^{*}$ and $\left(W_{\text {tot }}-W_{u}\right) / W_{\text {tot }}$, including data from finite element calculations for conical indenters and experimental results for a few materials using pyramidal indenters. (a) For $0.0<\left(W_{\text {tot }}\right.$ 
The functional dependence of $\Pi_{\theta}$ is displayed in Fig. 3. The subscript, $\theta$, denotes a possible dependence on indenter angle, since Eq. (8) and Fig. 3 were obtained for a particular indenter angle. Nevertheless, Eq. (8) shows that, for a given indenter angle, there is an approximate one-to-one correspondence between $H / E^{*}$ and $1-W_{u} / W_{\text {tot }}$. Consequently, the value $H / E^{*}$ may be obtained from the measurement of $W_{u}$ and $W_{\text {tot }}$, which can be determined readily by simple numerical integration based on force and displacement measurements.

The ratio of hardness to elastic modulus, $H / E^{*}$, is of significant interest in both tribology and fracture mechanics. This ratio multiplied by a geometric factor is the "plasticity index" which describes the deformation properties of a rough surface in contact with a smooth surface. ${ }^{13}$ When the plasticity index is much less than unity, the deformation of asperities is likely to be entirely elastic. When it is significantly greater than one, the deformation is predominantly plastic. In fracture mechanics, the quantity of $H / E^{*}$ appears in various fracture toughness equations. ${ }^{14} \mathrm{~A}$ few methods have been proposed to obtain this ratio, including that by Marshall et al. ${ }^{15}$ from the measurement of the diagonals of Knoop indentation. The present method provides an alternative method for measuring $H / E^{*}$ on micro- and nanometer scale for both metals and ceramics.

In the following, we show that it is possible to obtain both $H$ and $E^{*}$ using Eq. (8) and the well-known relationship,

$$
\left.\frac{d F}{d h}\right|_{h=h_{m}}=\frac{2}{\sqrt{\pi}} E^{*} \sqrt{A},
$$

where $d F /\left.d h\right|_{h=h_{m}}$ is the initial unloading slope and $A$ is the projected contact area evaluated at $h_{m}$. Equation (9) was initially derived for indentation into elastic solids. ${ }^{16}$ Using the infinitesimal theory of continuum mechanics, we have recently generalized it to the initial unloading in elasticplastic solids using indenters with axisymmetric smooth profiles. ${ }^{17}$ Furthermore, we have shown analytically that Eq. (9) holds true even for materials with work hardening and initial stress. ${ }^{17}$

Using Eq. (9) and the definition of hardness under full load, we obtain

$$
\frac{H}{E^{* 2}}=\frac{4}{\pi} \frac{F}{(d F / d h)^{2}},
$$

in which $F$ and $d F / d h$ are both evaluated at $h_{m}$. Therefore, the ratio of $H / E^{* 2}$ can be obtained from instrumented indentation experiments. Consequently, both $H$ and $E^{*}$ can, in principle, be obtained by combining Eqs. (8) and (10), i.e.,

$$
\begin{aligned}
& H=\frac{\pi}{4} \Pi_{\theta}^{2}\left(\frac{W_{\mathrm{tot}}-W_{u}}{W_{\mathrm{tot}}}\right) \frac{(d F / d h)^{2}}{F}, \\
& E^{*}=\frac{\pi}{4} \Pi_{\theta}\left(\frac{W_{\mathrm{tot}}-W_{u}}{W_{\mathrm{tot}}}\right) \frac{(d F / d h)^{2}}{F} .
\end{aligned}
$$

Several experimental data points obtained using Berkovich diamond indenters from literature for $\mathrm{Cu},{ }^{18} \mathrm{~W}, \mathrm{Al}$, fused silica, and sapphire ${ }^{5}$ are also included in Fig. 3. The finite elastic constants of the diamond indenter is taken into acDownloaded 06 Jun 2008 to 159.226 .100 .195 . Redistribution subje count by using the reduced modulus commonly defined as: $1 / E^{*}=\left(1-\nu^{2}\right) / E+\left(1-\nu_{i}^{2}\right) / E_{i}$, where $E_{i}=1140 \mathrm{GPa}$ and $\nu_{i}=0.07$ are the Young's modulus and Poisson's ratio of the diamond indenter, respectively. Since these experimental points lie approximately on the same curve as the finite element results, Eq. (8) seems thus applicable to indentation using these pyramidal indenters. Furthermore, since Eq. (9) has been shown to hold true for the pyramidal indenters with small $(<3 \%)$ corrections, ${ }^{19}$ it appears that Eq. (11) would, therefore, be applicable to indentation using pyramidal indenters.

We would like to re-emphasize that the relationship between $H / E^{*}$ and the work of indentation shown in Figs. 3(a) and 3(b) as well as Eq. (8) is an approximate one based on observation of a large number of finite element calculations and a few experimental data points. Nevertheless, it appears to be sufficient for estimating $H / E^{*}$ to within about $15 \%$ using the mean value at each $\left(W_{\text {tot }}-W_{u}\right) / W_{\text {tot }}$ shown in Fig. 3 . The accuracy may be improved by noticing the workhardening dependency especially when $H / E^{*}$ is small [Fig. $3(\mathrm{~b})$ ], provided that the work-hardening exponent, $n$, is known. Alternatively, the Oliver and Pharr procedure may be used to estimate the contact area to determine $H$ and $E^{*}$, since this method is reasonable for materials that moderately work harden. ${ }^{20}$ However, we encourage additional experiments, as well as modeling efforts, to truly ascertain the accuracy of the method.

The authors would like to thank W. J. Meng, S. J. Harris, G. L. Eesley, J. R. Smith, L. C. Lev, W. J. Baxter, D. D. Snyder, and K. C. Taylor for helpful discussions. Correspondences with Professor D. Tabor and Professor G. M. Pharr have also been valuable.

${ }^{1}$ D. Tabor, Philos. Mag. A 74, 1207 (1996).

${ }^{2}$ J. B. Pethica, R. Hutchings, and W. C. Oliver, Philos. Mag. A 48, 593 (1983).

${ }^{3}$ D. Stone, W. R. LaFontaine, P. Alexopoulos, T. W. Wu, and C.-Y. Li, J. Mater. Res. 3, 141 (1988)

${ }^{4}$ B. Bhushan, A. Kulkarni, W. Bonin, and J. Wyrobek, Philos. Mag. A 74, 1117 (1996)

${ }^{5}$ W. C. Oliver and G. M. Pharr, J. Mater. Res. 7, 1564 (1992).

${ }^{6}$ M. F. Doener and W. D. Nix, J. Mater. Res. 1, 601 (1986).

${ }^{7}$ Y.-T. Cheng and C.-M. Cheng, "Scaling laws in conical indentation of elastic-perfertly plastic solids," GM Research and Developmetn Center Publication R\&D-8689, June 23, 1997; Int. J. Solids Struct. (in press).

${ }^{8}$ Y.-T. Cheng and C.-M. Cheng, "A scaling approach to conical indentation in elastic-plastic solids with work-hardening," GM Research and Development Center Publication R\&D-8741, November 18, 1997; J. Appl Phys. (in press).

${ }^{9}$ J. Lubliner, Plasticity Theory (Macmillan, New York, 1990).

${ }^{10}$ G. Dieter, Mechanical Metallurgy, 2nd ed. (McGraw-Hill, New York, 1976).

${ }^{11}$ A. K. Bhattacharya and W. D. Nix, Int. J. Solids Struct. 24, 881 (1988).

${ }^{12}$ Y.-T. Cheng and C.-M. Cheng, Philos. Mag. Lett. 77, 39 (1998).

${ }^{13}$ J. A. Williams, Engineering Tribology (Oxford University, Oxford, 1994).

${ }^{14}$ I. J. McColm, Ceramic Hardness (Plenum, New York, 1990).

${ }^{15}$ D. B. Marshall, T. Noma, and A. G. Evans, J. Am. Ceram. Soc. 65, C-175 (1982).

${ }^{16}$ G. M. Pharr, W. C. Oliver, and F. R. Brotzen, J. Mater. Res. 7, 613 (1992).

${ }^{17}$ C.-M. Cheng and Y.-T. Cheng, Appl. Phys. Lett. 71, 2623 (1997).

${ }^{18}$ S. V. Hainsworth, H. W. Chandler, and T. F. Page, J. Mater. Res. 11, 1987 (1996)

${ }^{19}$ B. C. Hendrix, J. Mater. Res. 10, 255 (1995).

${ }^{20}$ A. Bolshakov and G. M. Pharr, J. Mater. Res. 13, 1049 (1998).

to AIP license or copyright; see http://apl.aip.org/apl/copyright.jsp 